\title{
Elevated fecal calprotectin levels are associated with severity of atopic dermatitis in children
}

\author{
SungChul Seo, ${ }^{1}$ So Hyun Ahn, ${ }^{2}$ Soohyun Ri, ${ }^{2}$ Yoonsun Yoon, ${ }^{2}$ Jung Hye Byeon, ${ }^{2}$ Seung Hyun Kim, ${ }^{3}$ Wonsuck Yoon, ${ }^{3}$ Young Yoo ${ }^{1,2,3}$
}

\begin{abstract}
Background: Recent data suggested that imbalance in gut microbiota and gastrointestinal inflammation are associated with the childhood allergic disease. Fecal calprotectin has been used for a non-invasive marker of gut inflammation.

Objective: The aim of this study was to investigate the relationships between fecal calprotectin level and the clinical severity of atopic dermatitis $(\mathrm{AD})$ in children.

Methods: We enrolled 65 subjects with AD. The concentration of calprotectin was measured in each subject's fecal sample.

Results: The geometric mean fecal calprotectin level of the total subjects was 33.1(10.1-108.9) $\mu \mathrm{g} / \mathrm{g}$. Among the 65 subjects,

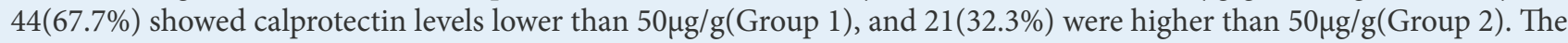
mean SCORAD index was significantly higher in Group 2 than Group $1(31.0 \pm 16.0$ vs $22.2 \pm 15.3$, p=0.046). The geometric mean serum total IgE levels was higher in Group 2 compared to Group 1(361.4[31.6-992.3]IU/mL vs 175.9[44.3-699.2]IU/ $\mathrm{mL}, \mathrm{p}=0.040)$. The mean blood eosinophils were significantly higher in Group 2 than in Group 1(497.7[239.8-1032.8]/ $\mu \mathrm{L}$ vs $281.5[121.5-652.0] / \mu \mathrm{L}, \mathrm{p}=0.034)$. The incidence of exposure to environmental tobacco smoke was significantly higher in Group 2 compared to Group $1(76.2 \%$ vs $47.7 \%$, $\mathrm{p}=0.036)$. Geometric mean fecal calprotectin level in severe AD was significantly higher than that of mild-to-moderate $\mathrm{AD}(66.7[13.5-330.3] \mu \mathrm{g} / \mathrm{g}$ vs $29.4[10.1-85.6] \mu \mathrm{g} / \mathrm{g}, \mathrm{p}=0.044)$. The fecal calprotectin level significantly correlated with the SCORAD index $(r=0.303, p=0.014)$.
\end{abstract}

Conclusions: Higher fecal calprotectin levels were observed in subjects with severe AD. Elevated fecal calprotectin level as a gastrointestinal inflammatory marker may associate with childhood AD. Measurement of fecal calprotectin might be useful for assessment of severity of childhood AD.

Keywords: Atopic dermatitis, Calprotectin, Children, Gut inflammation, Gut microbiota

\footnotetext{
From:

${ }^{1}$ Environmental Health Center, Korea University Anam Hospital ${ }^{2}$ Department of Pediatrics, College of Medicine, Korea University

${ }^{3}$ Allergy Immunology Center, Korea University, Seoul, Korea
}

\section{Introduction}

Atopic dermatitis $(\mathrm{AD})$ is a serious global disease associated with a modern lifestyle. ${ }^{1}$ This chronic inflammatory and pruritic skin disorder affects up to $20 \%$ of children. ${ }^{2}$ Various immune dysregulation, environmental factors, skin barrier dysfunction, and gut microbiota diversity have been emphasized as crucial pathogenesis in $\mathrm{AD}^{3}$ Although aberrant interactions exist between gastrointestinal inflammation, immune system, and allergic diseases, ${ }^{4-6}$ the nature of gut inflammation underlying $\mathrm{AD}$ remains unclear.

The hygiene hypothesis states that reduced exposure to microbes in early childhood affects the natural development of the immune system or immune tolerance, resulting in an
Corresponding author:

1. Young Yoo

Department of Pediatrics, College of Medicine, Korea University

73 Inchon-ro, Sungbuk-gu, Seoul 02841, Korea

Email: yoolina@korea.ac.kr

2. Wonsuck Yoon

Allergy Immunology Center, Korea University

73 Inchon-ro, Sungbuk-gu, Seoul 02841, Korea

Email: biokorea@korea.ac.kr

increased susceptibility to allergic diseases. ${ }^{7}$ The inflamed gut epithelium with impaired barrier function has been associated with various disorders, including AD. ${ }^{4}$ Although the direct cause and pathogenesis of $\mathrm{AD}$ are poorly defined, the concurrent dysbiosis in the gut microbiota has been observed in association with AD. ${ }^{8}$ Numerous studies also suggest that imbalance in the gut microbiota and subsequent gut inflammation are associated with allergic diseases. ${ }^{5,6,8}$

Calprotectin is a protein abundant in neutrophils and 
monocytes. Elevated concentrations of calprotectin can be measured in plasma, urine, and feces when there is ongoing inflammation..$^{9,10}$ Increased calprotectin levels have been found in chronic inflammatory bowel disease or allergic diseases. ${ }^{10-12}$ Fecal calprotectin is a potentially important clinical test as a marker of gastrointestinal tract inflammation and has been increasingly used as a non-invasive marker for the diagnosis of bowel inflammation or gastrointestinal allergic diseases..$^{10,11,13}$

Although many studies have reported the relationship between fecal calprotectin and gastrointestinal inflammation or allergic diseases, calprotectin has rarely been studied in AD. The aims of this study were to examine fecal calprotectin levels in children with $\mathrm{AD}$ and to investigate its relationship with the severity of AD.

\section{Materials and Methods \\ Study subjects}

Sixty-five subjects with $\mathrm{AD}$ aged 3 to 18 years old were recruited for this study from outpatients who attended the Allergy Clinic of Korea University Anam Hospital. The diagnosis of $\mathrm{AD}$ was based on the criteria of Hanifin and Rajka $^{14}$ and the SCORing of Atopic Dermatitis (SCORAD) index for assessing the severity of $\mathrm{AD}$ was evaluated by a pediatric allergist (Yoo Y). Among the selected subjects who met the diagnostic criteria of $\mathrm{AD}$, we excluded subjects aged less than 3 years to eliminate the possible effects of breast -feeding or food allergy manifested as AD. Subjects used topical steroids, topical antibiotics, or calcineurin inhibitors for treatment of $\mathrm{AD}$ at the time of enrollment. None of the subjects received systemic steroids, systemic antibiotics or probiotics within 1 month before stool sample collection. Subjects with a history of food allergy or other co-morbid allergic diseases such as rhinitis or asthma, or inflammatory bowel disease were excluded from the study. We defined food allergy based on the history of subject who experienced an immediate reaction following ingestion of a specific food and increased serum food-specific IgE levels. Rhinitis was defined on the basis of subject' history and rhinologic examination. Subject who had recurrent symptoms of sneezing, rhinorrhea, and nasal stuffiness or itching apart from common cold during the previous year was considered as having rhinitis. Subject who had typical symptoms suggestive of asthma, such as a history of episodic wheezing and/or dyspnea as well as reversible airflow obstruction and/or airway hyperresponsiveness assessed by methacholine bronchial challenge test was considered as having asthma. Subjects or their parents provided written informed consent for the study. The study protocol was approved by the Institutional Review Board of the Korea University Anam Hospital (No. AN16201).

\section{Questionnaires}

The subjects or their parents completed the questionnaire which included items of age, sex, family history of allergic diseases, detailed history after food ingestion, the mode of delivery, birth weight, breastmilk feeding, and exposure to environmental tobacco smoke (ETS).

\section{Measurement of fecal calprotectin concentration}

The stool samples were obtained from 65 subjects at the time of enrollment. If subject used probiotics, stool sample was collected at least 4 weeks after quit them. Stool was collected in a screw-capped plastic container and sent to the laboratory on the same day. An extraction buffer containing citrate and urea was added on the stool sample. The samples were mixed by a vortex method for 30 seconds and homogenized for 25 minutes. One milliliter of the homogenate was transferred to a tube and centrifuged for 20 minutes. Calprotectin was analyzed using a quantitative enzyme-linked immunosorbent assay according to the manufacturer's instructions (Calprest, Eurospital SpA, Trieste, Italy). Calprotectin was expressed as micrograms per gram of feces. We divided the subjects into 2 groups according to their fecal calprotectin levels. Subjects whose calprotectin levels were lower than healthy subject's cut off level $(50 \mu \mathrm{g} / \mathrm{g})^{15}$ were classified as Group 1 and higher than $50 \mu \mathrm{g} / \mathrm{g}$ were designated as Group 2.

\section{MAST-immunoblot assay}

The MAST-immunoblot assay was performed using a food panel of the AdvanSure Allergy Screen kit (LG Life Sciences, Seoul, Korea), which consists of 23 types of food allergens (soybean, milk, cheese, egg white, crab, shrimp, tuna, codfish, salmon, mackerel, pork, chicken, beef, citrus mix, peach, tomato, wheat, buckwheat, rice, barley meal, garlic, onion, and peanut), 6 types of pollen allergens (birch-alder mix, oak, rye, mugwort, ragweed, and Japanese hop), and 11 types of aeroallergens (yeast, Alternaria, Aspergillus, Cladosporium, Candida, cat, dog, cockroach, Dermatophagoides pteronyssinus, Dermatophagoides farinae, and Acarus siro). The test was performed according to the manufacturer's recommendations. The results were classified into 7 levels ranging from class 0 to class 6 . Reactions more than or equal to class $2(\geq 0.7 \mathrm{IU} / \mathrm{mL})$ were considered positive.

\section{Statistical analysis}

Data are presented as mean $\pm \mathrm{SD}$ or geometric mean (range of $1 \mathrm{SD}$ ) as appropriate. Serum total IgE levels, blood eosinophils, and fecal calprotectin levels were log transformed before statistical analysis. Differences between the 2 groups were tested using Student's $t$ test. Correlation analyses were performed using Pearson's correlation coefficients. All statistical analyses were performed using SPSS 20 for Windows. A p value of $<0.05$ was considered significant.

\section{Results}

Among the 65 subjects with AD, 44 (67.7\%) showed calprotectin levels lower than $50 \mu \mathrm{g} / \mathrm{g}$ (Group 1) and 21 (32.3\%) were higher than $50 \mu \mathrm{g} / \mathrm{g}$ (Group 2). There was no significant difference in age, sex, body mass index (BMI), and birth weight between the 2 groups. The prevalence of the family history of allergic diseases, the mode of delivery, or breastfed, was not different between the 2 groups, too. The incidence of exposure to ETS was significantly higher in Group 2 compared to Group $1(76.2 \%$ vs $47.7 \%, \mathrm{p}=0.036)$. Twenty-one (32.3\%) subjects used topical steroids, 3 (4.6\%) used topical antibiotics and 2 (3.1\%) used calcineurin inhibitors for $\mathrm{AD}$ treatment at the time of 
enrollment. In fact, 23 (35.4\%) subjects with positive specific IgE to food allergens were included in this study. Positive sensitization rates to food allergens were not different between the 2 groups ( $34.1 \%$ vs $38.1 \%, p=0.787$ ) (Table 1 ). The geometric means (range of 1SD) of fecal calprotectin levels were not different between the positive- and negative food specific-IgE groups (36.6 [11.7-114.4 $\mu \mathrm{g} / \mathrm{g}]$ vs $31.5[9.21-107.8 \mu \mathrm{g} / \mathrm{g}]$, $\mathrm{p}=0.625)$.

Table 1. Characteristics of the subjects with atopic dermatitis

\begin{tabular}{lccc}
\hline Parameters & $\begin{array}{c}\text { Group 1 } \\
(\mathbf{N}=\mathbf{4 4})\end{array}$ & $\begin{array}{c}\text { Group 2 } \\
\mathbf{( N = 2 1 )}\end{array}$ & p-value \\
\hline Age, years & $9.0 \pm 3.2$ & $8.0 \pm 2.6$ & 0.182 \\
\hline Boys, N(\%) & $14(31.8)$ & $9(42.9)$ & 0.417 \\
\hline Body mass index (kg/m $\left.{ }^{2}\right)$ & $17.8 \pm 2.9$ & $18.5 \pm 3.2$ & 0.348 \\
\hline Family history of allergy, N(\%) & $35(79.5)$ & $17(81.0)$ & 0.999 \\
\hline Cesarean section, N(\%) & $11(25.0)$ & $9(43.9)$ & 0.212 \\
\hline Birth weight, kg & $3.25 \pm 0.47$ & $3.18 \pm 0.49$ & 0.567 \\
\hline Prematurity, N(\%) & $2(4.5)$ & $2(9.5)$ & 0.589 \\
\hline Breast milk feeding, N(\%) & $30(68.2)$ & $13(61.9)$ & 0.780 \\
\hline $\begin{array}{l}\text { Environmental tobacco smoke, } \\
\text { N(\%) }\end{array}$ & $21(47.7)$ & $16(76.2)$ & 0.036 \\
\hline $\begin{array}{l}\text { Sensitization to food allergen, } \\
\text { N(\%) }\end{array}$ & $15(34.1)$ & $8(38.1)$ & 0.787 \\
\hline $\begin{array}{l}\text { Sensitization to aeroallergen, } \\
\text { N(\%) }\end{array}$ & $22(50.0)$ & $14(66.7)$ & 0.287 \\
\hline
\end{tabular}

Data are expressed as mean $\pm \mathrm{SD}$

The geometric mean (range of $1 \mathrm{SD}$ ) fecal calprotectin level was $16.6(10.2-27.1) \mu \mathrm{g} / \mathrm{g}$ in Group 1 and 142.6 (60.9-333.6) $\mu \mathrm{g} / \mathrm{g}$ in Group $2(\mathrm{p}<0.001)$. The mean \pm SD SCORAD index was significantly higher in Group 2 than Group 1 (31.0 \pm 16.0 vs $22.2 \pm 15.3, p=0.046)$. The geometric mean (range of $1 \mathrm{SD}$ ) serum total IgE levels were higher in Group 2 compared to Group 1 (361.4 [131.6-992.3] IU/mL vs 175.9 [44.3-699.2] IU/mL, $\mathrm{p}=0.040)$. The peripheral blood eosinophils were significantly higher in Group 2 than in Group 1 (497.7 [239.8-1032.8] $/ \mu \mathrm{L}$ vs $281.5[121.5-652.0] / \mu \mathrm{L}, \mathrm{p}=0.010)$. There was no significant difference in the mean age at the time of $\mathrm{AD}$ diagnosis between Groups 1 and 2 (2.3 \pm 2.2 years vs $2.1 \pm 1.8$ years) (Table 2 ).

We compared fecal calprotectin levels in $\mathrm{AD}$ subjects according to the severity of disease. Fifty-five (84.6\%) subjects belonged to the mild-to-moderate AD (SCORAD < 40) and 10 subjects $(15.4 \%)$ belonged to the severe AD (SCORAD $\geq 40$ ). The geometric mean (range of $1 \mathrm{SD}$ ) fecal calprotectin levels in severe $\mathrm{AD}(66.7$ [13.5-330.3] $\mu \mathrm{g} / \mathrm{g})$ was significantly higher than that in mild-to-moderate $\mathrm{AD}(29.4$ [10.1-85.6] $\mu \mathrm{g} / \mathrm{g}, \mathrm{p}=0.044)$. The serum IgE levels, sensitization rates to aeroallergens, blood eosinophils were higher in subjects with severe AD (Table 3). We further analyzed the 5 subjects who had a high SCORAD index $(\geq 40)$ but a low fecal calprotectin level $(<50 \mu \mathrm{g} / \mathrm{g})$. The mean \pm SD age $(12.4 \pm 5.2$ years $)$ was older and the duration of
Table 2. SCORAD indices and fecal calprotectin levels in subjects with atopic dermatitis

\begin{tabular}{lccc}
\hline Parameters & $\begin{array}{c}\text { Group 1 } \\
(\mathbf{N}=\mathbf{4 4})\end{array}$ & $\begin{array}{c}\text { Group 2 } \\
\mathbf{( N = 2 1 )}\end{array}$ & p-value \\
\hline Age at diagnosis, years & $2.3 \pm 2.2$ & $2.1 \pm 1.8$ & 0.635 \\
\hline $\begin{array}{l}\text { Duration of disease, } \\
\text { years }\end{array}$ & $6.7 \pm 3.9$ & $5.9 \pm 2.9$ & 0.404 \\
\hline SCORAD & $22.2 \pm 15.3$ & $31.0 \pm 16.0$ & 0.046 \\
\hline Blood eosinophils, $/ \mu \mathrm{L}$ & 281.5 & 497.7 & 0.010 \\
\hline Serum total IgE, IU/mL & $171.5-652.0)$ & $(239.8-1032.8)$ & \\
\hline Fecal calprotectin, $\mu \mathrm{g} / \mathrm{g}$ & $(44.3-699.2)$ & $(131.6-992.3)$ & 0.040 \\
\hline Hemoglobin, g/dL & 16.6 & 142.6 & $<0.001$ \\
\hline ESR, mm/hr & $13.1 \pm 0.27 .1)$ & $(60.9-333.6)$ & \\
\hline C-reactive protein, mg/L & $1.6 \pm 5.2$ & $12.7 \pm 0.7$ & 0.141 \\
\hline Vitamin D, ng/mL & $34.3 \pm 8.9$ & $34.6 \pm 8.3$ & 0.914 \\
\hline Zn, $\mu$ g/dL & $75.0 \pm 9.3$ & $75.3 \pm 8.2$ & 0.897 \\
\hline
\end{tabular}

Data are expressed as mean \pm SD or geometric mean (range of $1 \mathrm{SD}$ ). Group 1, calprotectin $<50 \mu \mathrm{g} / \mathrm{g}$; Group 2, calprotectin $\geq 50 \mu \mathrm{g} / \mathrm{g}$. Abbreviations: SCORAD, scoring of atopic dermatitis; ESR, erythrocyte sedimentation rate

Table 3. Comparison of characteristics according to the severity of atopic dermatitis

\begin{tabular}{|c|c|c|c|}
\hline Parameters & $\begin{array}{l}\text { Mild-to-moderate } \\
\qquad \operatorname{AD}(\mathrm{N}=55)\end{array}$ & $\begin{array}{c}\text { Severe } \\
\text { AD }(\mathrm{N}=10)\end{array}$ & p-value \\
\hline Age, years & $8.3 \pm 2.7$ & $10.4 \pm 4.5$ & 0.189 \\
\hline Boys, N(\%) & $36(65.5)$ & $6(60)$ & 0.733 \\
\hline $\begin{array}{l}\text { Family history of } \\
\text { allergy, } N(\%)\end{array}$ & $44(80)$ & $8(80)$ & 1.000 \\
\hline Age at onset, years & $2.4 \pm 2.1$ & $1.2 \pm 1.6$ & 0.056 \\
\hline $\begin{array}{l}\text { Duration of disease, } \\
\text { years }\end{array}$ & $5.9 \pm 3.2$ & $9.2 \pm 4.6$ & 0.008 \\
\hline $\begin{array}{l}\text { Sensitization to food } \\
\text { allergen, } N(\%)\end{array}$ & $19(34.5)$ & $4(40)$ & 0.733 \\
\hline $\begin{array}{l}\text { Sensitization to } \\
\text { aeroallergen, } \mathrm{N}(\%)\end{array}$ & $27(49.1)$ & $9(90)$ & 0.034 \\
\hline SCORAD & $19.6 \pm 9.9$ & $53.0 \pm 11.8$ & $<0.001$ \\
\hline $\begin{array}{l}\text { Blood eosinophils, } \\
/ \mu \mathrm{L}\end{array}$ & $\begin{array}{c}311.1 \\
(137.0-706.3)\end{array}$ & $\begin{array}{c}550.0 \\
(242.3-1248.9)\end{array}$ & 0.047 \\
\hline $\begin{array}{l}\text { Serum total IgE, IU/ } \\
\mathrm{mL}\end{array}$ & $\begin{array}{c}183.1 \\
(50.9-658.5)\end{array}$ & $\begin{array}{c}632.7 \\
(239.8-1669.0)\end{array}$ & 0.005 \\
\hline $\begin{array}{l}\text { Fecal calprotectin, } \\
\mu \mathrm{g} / \mathrm{g}\end{array}$ & $\begin{array}{c}29.4 \\
(10.1-85.6)\end{array}$ & $\begin{array}{c}66.7 \\
(13.5-330.3)\end{array}$ & 0.044 \\
\hline
\end{tabular}

Data are expressed as mean $\pm \mathrm{SD}$ or geometric mean (range of $1 \mathrm{SD}$ ). Abbreviations: SCORAD, scoring of atopic dermatitis 
disease $(11.0 \pm 5.5$ years $)$ was much longer in the 5 subjects than in the remaining subjects. Other parameters were not significantly different in these 5 subjects.

We also analyzed possible correlations between fecal calprotectin levels and various biologic parameters of $\mathrm{AD}$. The fecal calprotectin level significantly correlated with SCORAD index ( $r=0.303, p=0.014$, Figure), but showed no statistical significance with other parameters (Table 4).

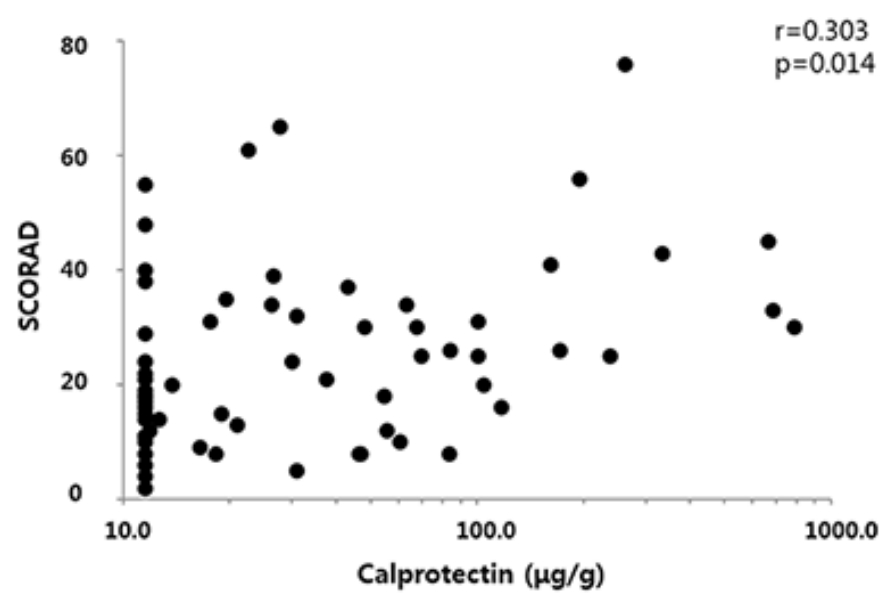

Figure. Correlation between fecal calprotectin levels and SCORAD indices in subjects with atopic dermatitis.

Table 4. Correlations between fecal calprotectin levels and other parameters

\begin{tabular}{lcc}
\hline Parameters & $\mathbf{r}$ & p-value \\
\hline Duration of atopic dermatitis, years & -0.137 & 0.275 \\
SCORAD & 0.303 & 0.014 \\
\hline Blood eosinophils, $/ \mu \mathrm{L}$ & 0.135 & 0.283 \\
\hline Serum IgE, IU/mL & 0.129 & 0.307 \\
\hline Hemoglobin, g/dL & -0.076 & 0.547 \\
\hline ESR, mm/hr & 0.017 & 0.891 \\
\hline C-reactive protein, mg/L & -0.022 & 0.861 \\
\hline Vitamin D, ng/mL & 0.058 & 0.644 \\
\hline Zn, $\mu$ g/dL & 0.184 & 0.142 \\
\hline
\end{tabular}

Abbreviations: SCORAD, scoring of atopic dermatitis; ESR, erythrocyte sedimentation rate

\section{Discussion}

The present study demonstrated that children with high fecal calprotectin levels had more severe AD assessed by the SCORAD index. Children with elevated fecal calprotectin levels also showed higher blood eosinophils and IgE levels. Several laboratory parameters have been used to assess disease severity in children with $\mathrm{AD}$ but, there was no objective non -invasive severity scale until now. Thus, simple and non-invasive tests are needed as markers of disease severity in children with $\mathrm{AD}$. In the present study, we showed that the measurement of fecal calprotectin might be useful for assessment of severity of
AD. Fecal calprotectin has been suggested to be as a simple and reproducible marker to evaluate the inflammatory status of the gastrointestinal tract and to monitor infants with cow's milk protein allergy. ${ }^{16-17}$

In the present study, a reference calprotectin value of a 50 $\mu \mathrm{g} / \mathrm{g}$ in feces is used as a cut-off level according to the study by Fagerberg et al. ${ }^{15}$ Fecal calprotectin concentrations seem to be age-dependent in infancy however, healthy children aged between 4-17 years apparently exhibit constant fecal calprotectin excretions similar to those of adults. ${ }^{15} \mathrm{AD}$ is one of the most common clinical manifestations of food allergy and may not be completely excluded from this study. Although we did not exclude patients with food allergy by food challenge, we tried not to include subjects who experienced an immediate reaction including $\mathrm{AD}$ flaring after ingestion of offending food. Up to two-thirds of infants with moderate-to-severe AD show sensitization to food allergens in the first 2 years of life however, only a minor proportion of sensitized infants have concomitant IgE-mediated food allergy. ${ }^{18}$ Thus, we excluded subjects aged less than 3 years to eliminate the possible effects of food allergy.

Increased levels of fecal calprotectin have been reported in children with inflammatory bowel disease or food allergy. ${ }^{10,19,20}$ In patients with inflammatory bowel disease, the fecal calprotectin level closely related with mucosal gastrointestinal inflammation. ${ }^{16}$ Fecal calprotectin levels were elevated and used to monitor mucosal healing in patients with ulcerative colitis as well. ${ }^{19}$ Thus, fecal calprotectin has been considered a sensitive marker of mucosal healing in gut inflammation. Compared with those for inflammatory bowel or autoimmune diseases, fecal calprotectin data on allergic diseases are poorly investigated. In a recently published data, ${ }^{17}$ fecal calprotectin levels were higher in children with non-IgE mediated cow's milk protein allergy than in controls. In addition, high fecal calprotectin levels were found in children with egg allergy. ${ }^{20}$ The fecal calprotectin levels were almost twice higher in children with challenge-proved food allergy than in those with negative challenge results. ${ }^{20}$ Thus, it could be useful for the diagnosis of a certain allergic diseases. However, only a few studies have addressed elevated calprotectin levels in the skin or serum in $\mathrm{AD} .{ }^{12,21}$ Orivuori et al. ${ }^{22}$ demonstrated that infants with high fecal calprotectin levels at the age of 2 months had an increased risk of developing $\mathrm{AD}$ by age 6 . They concluded that imbalance of the gut microbiota and intestinal inflammation in early life was associated with later development of AD.

The gut immune system is an important regulator of immune-mediated diseases, such as allergies. ${ }^{4}$ Numerous investigators have shown that changes in the gut microbiota and intestinal inflammation have been associated with the development of allergic diseases. ${ }^{6,8,13,23}$ The gut microbiota controls intestinal inflammation, which further modulates the development of the systemic immune system. ${ }^{8,23}$ Altered gut microbiota induces epithelial damage resulting in increased intestinal inflammation, altered gut permeability and immunological balance, which affect the development of allergic diseases. ${ }^{8,13,23}$ The damaged epithelium with increased permeability might allow for the passage of allergens. ${ }^{24}$ Prolonged enhanced permeability and increased exposure to allergens might result in the subsequent development of atopic eczema. ${ }^{6,24}$ Several studies have revealed that infants 
with eczema exhibit alterations in the relative levels of beneficial and potentially harmful bacteria compared to healthy infants. ${ }^{5,6}$ Although specific molecules produced by commensal bacteria that trigger inflammatory responses in the gut are still not well-characterized, numerous microbiota-associated molecules, including short chain fatty acid (SCFA), are known to regulate inflammatory responses through the induction of immune cells. ${ }^{25}$ Altered gut microbiota and subsequently reduced production in SCFA can be implicated in children with $\mathrm{AD} .{ }^{8}$ The gut microbiota appears to play an important role in controlling not only gut specific but also distant immune responses.

It is likely that elevated fecal calprotectin plays a pathophysiological role in the inflammatory process in gut. Some researchers investigated the link between gut microbiota and fecal calprotectin as an inflammatory marker. Results of decreased fecal calprotectin after administration of probiotics for 4 weeks support that probiotics or live microorganisms may affect the host by supplying beneficial intestinal bacteria and provide health benefits. ${ }^{26}$ Interestingly, infants with low fecal calprotectin levels shows a low Lactobacillaceae abundance and a high percentage of Escherichia in feces, which suggests early colonization of the gut microbiota regulate intestinal inflammation. This further implies that diminished gastrointestinal inflammation and subsequent improved allergic responses by administration of probiotics. In the present study, we showed higher fecal calprotectin level in subjects with severe AD. However, whether fecal calprotectin directly contributes to the pathogenetic mechanisms in children with $\mathrm{AD}$ remains to be explored.

Meanwhile, previous studies have demonstrated that exclusively breastfed infants have higher fecal calprotectin levels..$^{22,27}$ This could be explained by higher microbial exposure via breast milk. However, we could not find different fecal calprotectin levels in breastfed subjects. It is thought that since our subjects were 3 to 18 years old, breastfed effect did not continue until that age. In the present study, the incidence of exposure to ETS was significantly higher in subjects with high fecal calprotectin levels compared to those with low fecal calprotectin levels. Few studies have been conducted to determine the relationship between exposure to ETS and fecal calprotectin levels yet. Only 1 study demonstrated that serum calprotectin levels were higher in children with ETS exposure. ${ }^{28}$ In that study, the researchers concluded that increased serum levels of calprotectin might be an indicator of inflammation related to ETS exposure. As children are much more prone to ETS than adults, it is believed that the effects of ETS on inflammation in children are more significant than in adults. Higher fecal calprotectin levels may not indicate the long-term exposure to ETS, but it could be at risk of long-term continuing inflammation. One thing to be clarified is that we collected data on ETS exposure at home by questionnaires instead of cotinine levels, which may have introduced some bias.

In the present study, we showed increased blood eosinophils and IgE levels in subjects with elevated calprotectin levels. However, no significant correlations were found among those 3 parameters. It is suggested that these parameters would be closely related with the clinical severity of $\mathrm{AD}$ but reflect different aspects of inflammatory responses in $\mathrm{AD}$.
This study has some limitations. First, we chose calprotectin level of $50 \mu \mathrm{g} / \mathrm{g}$ as a cut-off value. Although $50 \mu \mathrm{g} / \mathrm{g}$ was suggested as a cut-off value in healthy children, ${ }^{17}$ fecal calprotectin levels in children with $\mathrm{AD}$ were not definitely proposed. Secondly, we found no significant correlation between fecal calprotectin levels and other inflammatory markers of AD. Lastly, the diagnosis of food allergy was not made by food challenge.

The results of this study suggest that increased intestinal inflammation as measured by fecal calprotectin may be related to $\mathrm{AD}$ in children and useful for assessment of disease severity. Further prospectively designed randomized controlled studies are needed to confirm the role of calprotectin in monitoring the clinical severity of AD.

\section{Acknowledgement}

This study was supported in part by a grant of the Environmental Health Center for Childhood Asthma (20152016), Ministry of Environment, Korea, by a grant of the Basic Science Research Program through the National Research Foundation of Korea (NRF-2015R1C1A2A01055801, NRF-201 5R1D1A4A01019910) and by a grant of the Korea University to Wonsuck Yoon and Young Yoo.

\section{References}

1. Worldwide variation in prevalence of symptoms of asthma allergic rhinoconjunctivitis, and atopic eczema; ISAAC. Lancet. 1998;351:1225-32.

2. Nutten S. Atopic dermatitis: global epidemiology and risk factors. Ann Nutr Metab. 2015;66 (suppl 1):8-16.

3. Flohr C, Mann J. New insights into the epidemiology of childhood atopic dermatitis. Allergy. 2014;69:3-16.

4. MacDonald TT, Monteleone G. Immunity, inflammation, and allergy in the gut. Science. 2005;25:1920-5

5. Kosiewicz MM, Dryden GW, Chhabra A, Alard P. Relationship between gut microbiota and development of $\mathrm{T}$ cell associated disease. FEBS Lett. 2014; 17:4195-206.

6. West CE, Ryden P, Lundin D, Engstrand L, Tulic MK, Prescott SL. Gut microbiome and innate immune response patterns in IgE-associated eczema. Clin Exp Allergy. 2015;45:1419-29.

7. Wold AE. The hygiene hypothesis revised: is the rising frequency of allergy due to changes in the intestinal flora? Allergy. 1998;53 (46 Suppl):20-5.

8. Song H, Yoo Y, Hwang J, Na YC, Kim HS. Faecalibacterium prausnitzii subspecies-level dysbiosis in the human gut microbiome underlying atopic dermatitis. J Allergy Clin Immunol. 2016;137:852-60.

9. Johne B, Fagerhol MK, Lyberg T, Prydz H, Brandzaeg P, Naess-Andersen $\mathrm{CF}$, et al. Functional and clinical aspects of the myelomonocyte protein calprotectin. Mol Pathol. 1997;50:113-23.

10. Papi C, Fasci-Spurio F, Rogai F, Settesoldi A, Margagnoni G, Annese V. Mucosal healing in inflammatory bowel disease: treatment efficacy and predictive factors. Dig Liver Dis. 2013;45: 978-85.

11. Belizon CT, Paez EO, Claros AM, Sanchez IR, Gonzales AMR, Medialdea $\mathrm{RV}$, et al. Faecal calprotectin as an aid to the diagnosis of non-IgE mediated cow's milk protein allergy. An Pediatr (Barc). 2016;84:318-23.

12. Jin S, Park CO, Shin JU, Noh JY, Lee YS, Lee NR, et al. DAMP molecules S100A9 and S100A8 activated by IL-17A and house-dust mites are increased in atopic dermatitis. Exp Dermatol. 2014;23:922-41.

13. Merras-Salmio L, Kolho KL, Pelkonen AD, Kuitunen M, Makela MJ, Savilahti E. Markers of gut mucosal inflammation and cow's milk specific immunoglobulins in non-IgE cow's milk allergy. Clin Transl Allergy. 2014;4:8.

14. Hanifin JM, Rajka G. Diagnostic features of atopic dermatitis. Acta Derm Venereol (Stockh). 1980;2:44-7.

15. Fagerberg UL, Loof L, Merzoug RD, Hansson LO, Finkel Y. Fecal calprotectin levels in healthy children studied with an improved assay. J Pediatr Gastroenterol Nutr. 2003;37:468-72. 
16. Takashima S, Kato J, Hiraoka S, Nakarai A, Takei D, Inokuchi T, et al. Evaluation of mucosal healing in ulcerative colitis by fecal calprotectin vs. fecal immunochemical test. Am J Gastroenterol. 2015;110:873-80.

17. Beser OF, Sancak S, Erkan T, Kutlu T, Cokugras H, Cokugras FC. Can fecal calprotectin level be used as a marker of inflammation in the diagnosis and follow-up of cow's milk protein allergy? Allergy Asthma Immunol Res. 2014;6:33-8.

18. D’Auria E, Banderali G, Barberi S, Gualandri L, Pietra B, Riva E, et al. Atopic dermatitis: recent insight on pathogenesis and novel therapeutic target. Asian Pac J Allergy Immunol 2016;34:98-108.

19. Hosseini SV, Safarpour AR, Taghavi SA. Developing a novel risk-scoring system for predicting relapse in patients with ulcerative colitis: a prospective cohort study. Pak J Med Sci. 2015;31:1511-16.

20. Winberg A, Nagaeva O, Nagaev I, Lundell C, Arencibia I, Mincheva -Nilsson L, et al. Dynamics of cytokine mRNA expression and fecal biomarkers in school-children undergoing a double-blind placebo -controlled food challenge series. Cytokine. 2016;88:259-66.

21. Aochi S, Tsuji K, Sakaguchi M, Huh N, Tsuda T, Yamanishi K, et al. Markedly elevated serum levels of calcium-binding S100A8/A9 proteins in psoriatic arthritis are due to activated monocytes/macrophages. J Am Acad Dermatol. 2011;64:879-87.
22. Orivuori L, Mustonen K, de Goffau MC, Hakala S, Paasela M, Roduit C, et al. High level of fecal calprotectin at age 2 months as a marker of intestinal inflammation predicts atopic dermatitis and asthma by age 6. Clin Exp Allergy. 2015;45:928-39.

23. Noverr MC, Huffnagle GB. Does the microbiota regulate immune responses outside the gut? Trends Microbiol. 2004;12:562-8.

24. Pike MG, Heddle RJ, Boulton P, Turner MW, Atherton DJ. Increased intestinal permeability in atopic eczema. J Invest Dermatol. 1986;86:101-4.

25. Maslowski KM, Mackay CR. Diet, gut microbiota and immune responses. Nat Immunol. 2011;12:5-9.

26. Baldassarre ME, Laforgia N, Fanelli M, Laneve A, Grosso R, Lifschitz C Lactobacillus GG improves recovery in infants with blood in the stools and presumptive allergic colitis compared with extensively hydrolyzed formula alone. J Pediatr. 2010;156:397-401.

27. Dorosko S, MacKenzie T, Connor RI. Fecal calprotectin concentrations are higher in exclusively breastfed infants compared to those who are mixed-fed. Breastfeed Med. 2008;3:117-9.

28. Cobanoglu N, Dalkan C, Galip N, Tekguc H, Uncu M, Bahceciler NN. Is calprotectin a marker of tobacco smoke related inflammation?: a pilot study in children. Inhal Toxicol. 2012;24:486-96. 